\title{
Actividades Físicas en el Medio Natural. Incidencia en la formación del Profesorado y su enseñanza en la ESO
}

Physical Activities in the Natural Environment. Impact on teacher training and its teaching at Secondary Education

Miguel Hurtado Barroso; Déborah Sanabrias Moreno; María Sánchez Zafra; Javier Cachón Zagalaz

Universidad de Jaén. Departamento de Didáctica de la Expresión Musical, Plástica y Corporal

Autor de correspondencia: María Sánchez Zafra mszafra@ujaen.es 


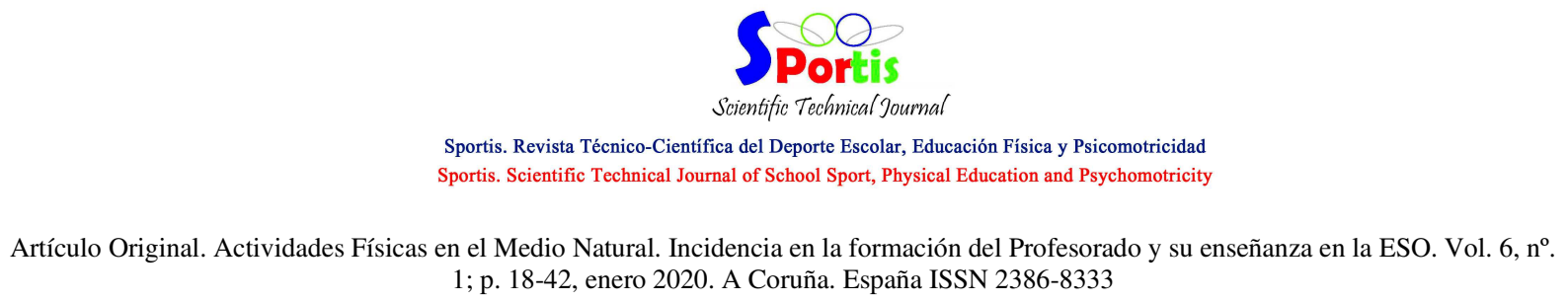

\title{
Resumen
}

El bloque de contenidos de Actividad Física en el Medio Natural (AFMN) es uno de los que menos se incluye en las programaciones de Educación Física (EF) en Enseñanza Secundaria Obligatoria (ESO), lo que, unido a la escasa preparación en dichos contenidos recibida en los estudios de formación de profesorado de EF, dificulta su enseñanzaaprendizaje en esta etapa educativa. Por tal motivo el objetivo de esta investigación es averiguar porque el profesorado de ESO no desarrolla o trabaja poco las AFMN. Para ello, se aplicó una entrevista semiestructurada de respuestas abiertas a trece docentes de diversas localidades andaluzas para averiguar cómo se trabaja el bloque de contenidos de AFMN. Los datos recogidos concluyen que la mayoría del profesorado trabaja la orientación y el senderismo como únicas actividades dentro del bloque $5^{\circ}$ (AFMN), argumentando la falta de medios, dificultad de organización de salidas y el riesgo que suponen este tipo de actividades.

\section{Palabras clave}

AFMN; Percepción del profesorado; ESO; Formación del profesorado

\begin{abstract}
The contents about the physical activity in the natural environment are one of the least visible in the educational programming for the Andalusian physical education teachers, mainly during the Obligatory Secondary Education. Therefore, we are trying to clarify the major reasons that lead teachers to ignore this type of content. For that purpose, a qualitative research is conducted by interviewing thirteen teachers from different Andalusian locations. The data collected conclude that most teachers work orienteering and hiking as the only activities within the fifth block, arguing the lack of means to support the activities, difficulty in organizing trips to the natural environment and the risk posed by these activities. In this research, we also try to simplify the difficulties encountered by teachers when physical activities are practiced in the natural environment. It is assumed that the training received due to the qualifications required to work as a physical education teacher does not train them to practice the activities in the natural environment.
\end{abstract}

\section{Keywords}

Activity in the natural environment; teacher's perception; Obligatory Secondary Education.

\section{Introducción}

A pesar de que la actividad física aporta múltiples beneficios a sus practicantes hay un gran número de personas que no la practican (Garrido-Méndez, et al.,2019). Actualmente, solo el 37\% de los adolescentes cumplen las recomendaciones de la OMS (2010) de realizar AF moderada-vigorosa durante 60 minutos diarios al menos 5 días a la semana. Por tanto, se podría afirmar que la asignatura de EF no cumple uno de sus principales objetivos "la

Para citar este artículo utilice la siguiente referencia: Hurtado, M.; Sanabrias, D.; Sánchez, Mª.; Cachón, J.. (2020). Actividades Físicas en el Medio

Natural. Incidencia en la formación del Profesorado y su enseñanza en la ESO. Sportis Sci J, 6 (1), 18-42.

DOI: https://doi.org/10.17979/sportis.2020.6.1.5769

http://revistas.udc.es/ 
Artículo Original. Actividades Físicas en el Medio Natural. Incidencia en la formación del Profesorado y su enseñanza en la ESO. Vol. 6, nº $1 ;$ p. 18-42, enero 2020. A Coruña. España ISSN 2386-8333

consolidación de hábitos regulares de práctica de AF saludable en la vida cotidiana y, también, ocupación activa del ocio y tiempo libre" por parte del alumnado (BOJA, 2016:265), a pesar de que es tarea del entorno escolar fomentar la consolidación de hábitos saludables (Rossi et al., 2018)

A pesar de las numerosas AF y deportivas que incluye el currículo vigente en ES, el informe EURYDICE de la Comisión Europea (2013) manifiesta que "se estima que hasta un $80 \%$ de niños y niñas en edad escolar únicamente participan en AF en la escuela”. Diversos estudios demuestran que más del $70 \%$ de los adolescentes españoles no siguen las recomendaciones establecidas por el OMS sobre el tiempo estimado como saludable de práctica de AF (Beltrán-Carrillo, Devís-Devís, \& Peiró-Velert, 2012; Oviedo, Sánchez, Castro, Calvo, Sevilla, Iglesias, \& Guerra, 2013; Ramos, Jiménez-Iglesias, Rivera, \& Moreno, 2016; Cuervo, Cachón, González, \& Zagalaz, 2017).

Estos datos reflejan que el profesorado de EF no consigue inculcar al alumnado la práctica de AF como saludable. Uno de los principales motivos puede ser que gran parte del alumnado encuentra las sesiones de EF aburridas y monótonas (Baena \& Graneros, 2015). Moral, Román-Palmero, López, Rosa, Pérez \& García (2019) afirman que el docente de EF es una pieza clave para conseguir involucrar a los alumnos en la práctica de AF fuera de la escuela, en su tiempo libre. Por ello se debe sorprender al alumnado y brindarle actividades que consigan una fuerte motivación intrínseca, con el fin de que practiquen AF no solo porque es bueno para la salud sino porque disfrutan practicándola, lo mismo que el niño juega, no para desarrollar sus habilidad motrices o sociales sino porque se divierte jugando. Son los padres, los docentes y los entrenadores los que han de conseguir fomentar los hábitos saludables en los niños y adolescentes (Tárrega, Alguacil, \& Parra, 2018)

El profesorado debe proporcionar al alumnado momentos en los que el aprendizaje se lleve también a cabo al aire libre, ofreciéndoles actividades que les permiten interactuar con espacios naturales (Wishart \& Rouse, 2018). La práctica de AF en la naturaleza aporta beneficios para la salud y favorece el contacto social (Castell, 2019). Baena \& Granero (2015), demostraron el poder motivacional que tiene la práctica de AFMN dentro de las clases de EF, por lo que plantear al alumnado este tipo de actividades puede favorecer el clima de Para citar este artículo utilice la siguiente referencia: Hurtado, M.; Sanabrias, D.; Sánchez, Mª ; Cachón, J.. (2020). Actividades Físicas en el Medio 


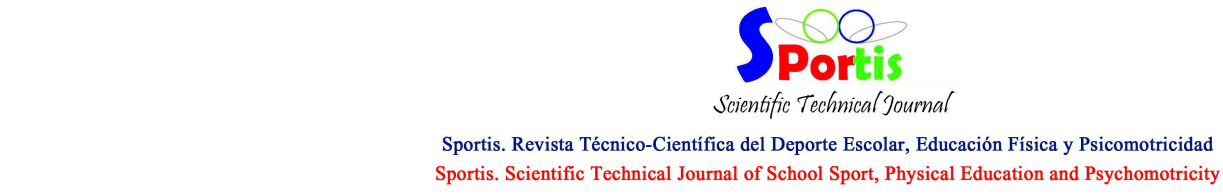

Artículo Original. Actividades Físicas en el Medio Natural. Incidencia en la formación del Profesorado y su enseñanza en la ESO. Vol. 6, nº $1 ;$ p. 18-42, enero 2020. A Coruña. España ISSN 2386-8333

trabajo de las sesiones. En este sentido, Gehris, Kress, \& Swalm (2010) en estudios similares, comprobaron que muchos de los alumnos, tras la incorporación de AFMN en las sesiones de EF decidieron practicar escalada en su tiempo libre, "debido, principalmente, a que se habían divertido mucho con estas actividades". Las AFMN han avanzado hasta llegar a posicionarse como una alternativa clara a los deportes tradicionales, siendo un modo de escapar de la rutina y probar nuevas experiencias en la naturaleza (López, Maneiro, Moral, Amatria, Diez, Barcala, \& Abelairas, 2019)

Las AFMN, además de su indudable carácter motivacional, aportan beneficios como el desarrollo de la condición física, y a nivel psicológico, el aumento de la autoestima, mejora del estado de ánimo, y liberación de estrés; a nivel social, el desarrollo de habilidades sociales y de cooperación y en el plano educativo, mejoras en el rendimiento académico, educación medioambiental, resolución de problemas, conciencia de los valores (Baena \& Granero, 2014; Ewert, 1987; Ruiz, García, \& Pierón, 2009). También favorecen la adquisición de competencias clave, tal y como afirman Méndez \& Fernández Río (2011) y Peñarrubia, Guillén, \& Lapetra (2011).

Los beneficios que aporta la actividad física en los menores se ven reforzados cuando esta se realiza en la naturaleza ya que se convierte en un entorno mediante el cual pueden liberar tensiones y autorregularse, así como promover un mejor desarrollo cognitivo (Roberts, Hinds \& Camic, 2019).

Es indudable que las salidas al Medio Natural favorecen la creación de un vínculo emocional con el mismo, lo que conlleva un aumento del sentido de responsabilidad hacia su respeto y cuidado del medio. Por ello, ya sea de forma programada o inconscientemente, en todas las sesiones de AFMN se trabaja la educación medioambiental. La realización de práctica de actividad física en el medio ambiente debe hacerse desde una perspectiva respetuosa ayudando a los participantes a vivir y sentir la necesidad de disfrutar la actividad física a través del cuidado y el respeto hacia la naturaleza (Santos-Pastor, Cañadas \& Martínez-Muñoz, 2019).

Para citar este artículo utilice la siguiente referencia: Hurtado, M.; Sanabrias, D.; Sánchez, Mª .; Cachón, J.. (2020). Actividades Físicas en el Medio Natural. Incidencia en la formación del Profesorado y su enseñanza en la ESO. Sportis Sci J, 6 (1), 18-42. DOI: https://doi.org/10.17979/sportis.2020.6.1.5769

http://revistas.udc.es/ 


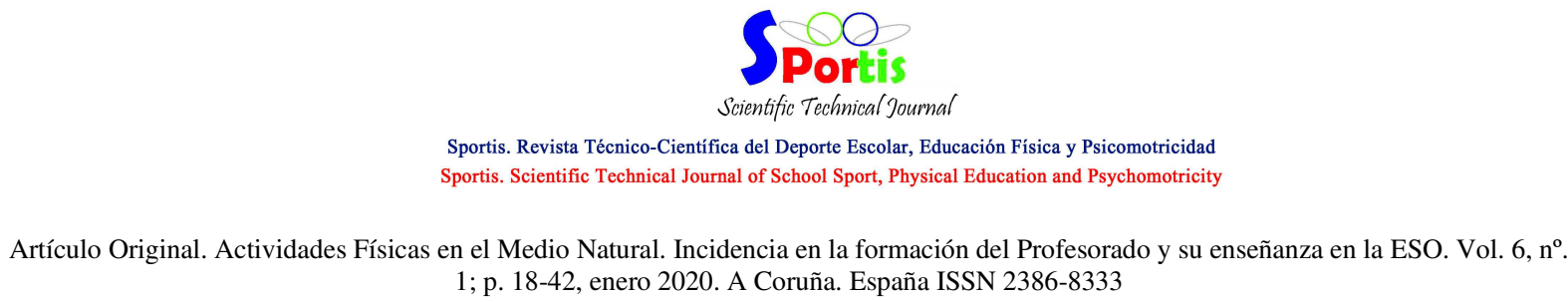

Sin embargo, al tratarse de estudiantes menores de edad, hay que considerar el riesgo que conllevan las AFMN. Riesgo, según el diccionario de la RAE significa: "Contingencia o proximidad de un daño", lo que aplicado a las AFMN, hace que haya que diferenciar entre riesgo objetivo y riesgo subjetivo. El primero, según Portell, Riba, \& Bayés (1997) es aquel que está sometido a diversos métodos de evaluación, basándose en datos estadísticos y cálculos matemáticos. El subjetivo es definido por Slovic (1987) como el que está relacionado con juicios intuitivos, dictados en función de "estructuras personales, cognoscitivas, emocionales, percepción y de motivación como con los ambientes sociales, culturales y políticos".

Se podría pensar que actividades como la espeleología suponen un alto riesgo objetivo, sin embargo, para Villolota (2017), en 2015 fueron 2.750 personas las rescatadas mientras practicaban deportes de montaña, acaparando el $42 \%$ los sujetos que practicaban senderismo. De igual modo, los datos de operaciones registradas por el GREIM (2014), detalladas por Sánchez Hernández (2017), muestran que en 2015, la práctica del senderismo es la que mayor número de rescates precisó (33\%) frente a la espeleología $(0.6 \%)$. Sin embargo, el senderismo, junto a la orientación, encabeza la lista de actividades más trabajadas en las aulas de los centros educativos (López-Prado, Álvarez García, \& Fernández-Miranda, 2015; Graneros, Baena, \& Martínez Molina, 2010; Sáez Padilla, Sierra, \& Tornero, 2017).

Estos datos no aportan información clara sobre el riesgo que supone la práctica de cada una de estas disciplinas, puesto que el número de senderistas es mucho más elevado que el de espeleólogos. Sin embargo, otro detalle importante, recogido por Sánchez Hernández (2017), es que el senderismo es el causante o precursor de estos accidentes. En el informe del GREIM (2014) se expone que el $40 \%$ de los accidentes son provocados por la sobreestimación de las posibilidades, el 38\% por falta de nivel técnico, el 30\% por escasez de planificación y el $28 \%$ por inexistencia de condición física.

Continuando con los beneficios que aportan las AFMN, tanto para el desarrollo físico, psicológico y sociológico como formativo (Peñarrubia, Guillén, \& Lapetra, 2015), autores como Robles, Giménez, \&Abad (2010) inciden en que este bloque de contenidos es el que menos visibilidad tiene en las sesiones de EF en ESO. Asimismo la bibliografía refleja que la

Para citar este artículo utilice la siguiente referencia: Hurtado, M.; Sanabrias, D.; Sánchez, Ma .; Cachón, J.. (2020). Actividades Físicas en el Medio Natural. Incidencia en la formación del Profesorado y su enseñanza en la ESO. Sportis Sci J, 6 (1), 18-42. 


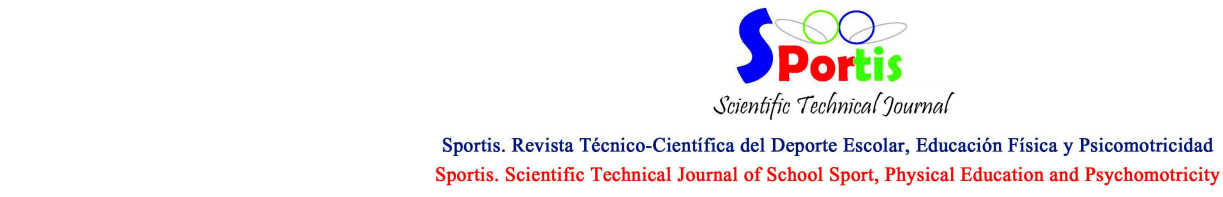

Artículo Original. Actividades Físicas en el Medio Natural. Incidencia en la formación del Profesorado y su enseñanza en la ESO. Vol. 6, nº $1 ;$ p. 18-42, enero 2020. A Coruña. España ISSN 2386-8333

orientación y el senderismo son prácticamente los únicos contenidos que se trabajan en relación a la práctica de AFMN (Granero, Baena, \& Martínez-Molina, 2010; Sáez-Padilla, 2005; y Torres, Monleón, Sánchez, Torres, \& Aranda, 2016). Lo justifican señalando la necesidad de usar material específico, la complejidad de realizar salidas al Medio Natural debido a la exigencia de modificar el horario escolar, la poca formación recibida por el profesorado con respeto a estas prácticas y, por último, el riesgo que supone la realización de dichas actividades.

Otros autores como López-Prado, Pardilla, \& Álvarez García (2013), presentan una serie de orientaciones didácticas para incluir el Surf en el contexto educativo sin la necesidad de realizar salidas al Medio Natural. Por tanto, cabe reflexionar sobre si realmente el profesor necesita un contexto concreto para dichas actividades o por el contrario no tiene la formación necesaria para impartir dichos contenidos adaptándolos al ámbito educativo.

El informe de Turismo Activo realizado en 2015 por la Asociación Nacional de Empresas de Turismo Activo (ANETA) revela que España cuenta con 4.245 empresas de turismo activo, perteneciendo a Andalucía el $20.71 \%$, lo que equivale a 879 empresas. El mismo informe muestra que más del 60\% de estas empresas se crearon entre los años 2004 y 2015. Estos datos reflejan una clara tendencia al alza, en cuanto a la práctica de AFMN por parte de la sociedad. Por ello, desde el área de EF, se debe aprovechar esta "moda" y mostrar al alumnado el amplio abanico de posibilidades que la AFMN ofrece, presentando diversos instrumentos para mejorar la salud, la calidad de vida y la ocupación activa del ocio y tiempo libre.

\section{Objetivos}

Se pretende averiguar los principales motivos por los que el $5^{\circ}$ bloque de contenidos de ESO recibe un trato discriminatorio. Partiendo de la hipótesis de que el profesorado de EF de ESO no ha adquirido durante su formación universitaria los conocimientos mínimos que le permitan trabajar este tipo de contenidos, se plantean como objetivos de esta investigación: 1 . Conocer cómo se trabaja el $5^{\circ}$ bloque de contenidos: AFMN en la etapa de ESO en los IES

Para citar este artículo utilice la siguiente referencia: Hurtado, M.; Sanabrias, D.; Sánchez, Mª .; Cachón, J.. (2020). Actividades Físicas en el Medio Natural. Incidencia en la formación del Profesorado y su enseñanza en la ESO. Sportis Sci J, 6 (1), 18-42. DOI: https://doi.org/10.17979/sportis.2020.6.1.5769

http://revistas.udc.es/ 
Artículo Original. Actividades Físicas en el Medio Natural. Incidencia en la formación del Profesorado y su enseñanza en la ESO. Vol. 6, nº 1 ; p. 18-42, enero 2020. A Coruña. España ISSN 2386-8333
\end{abstract}

andaluces 2. Averiguar las causas que llevan al profesorado a dar menor relevancia a estos contenidos en sus programaciones.

\title{
Material y métodos
}

\section{Diseño y participantes}

Se plantea un estudio de casos en la Comunidad Autónoma de Andalucía, utilizando la entrevista semiestructurada individual, preestableciendo una serie de preguntas abiertas, situación en la que el entrevistador pregunta a todos los participantes las mismas cuestiones con una serie limitada de categoría de respuesta (Rincón, Latorre, \& Sans, 1995).

\section{Muestra}

La muestra ha estado conformada por trece profesores de EF en activo, pertenecientes a la educación pública y concertada de diversos centros andaluces, tanto de zonas urbanas (7), como rurales (6) que respondieron a la entrevista de entre los 37 a quienes se les envió. Un 69.2\% de los profesores gozaba de más de 10 años de experiencia docente, un $7.7 \%$ tenía una experiencia de 5 a 10 años y un 23.1\% un máximo de 5 años. Más de limitad de los sujetos (63.7\%) se licencia/gradúa en la Universidad de Granada, frente a un 18.2\% que finalizó sus estudios en la Universidad Hispalense de Sevilla. Por último, de los sujetos entrevistados, un 9.1\% obtuvo su titulación en la Universidad sevillana Pablo de Olavide, compartiendo porcentaje de representación con la Politécnica de Madrid.

\section{Instrumentos y procedimiento}

En un primer momento, se realizó una revisión de la literatura, para conocer el estado de la cuestión de la formación inicial del profesorado de EF, así como para encontrar cuestionarios e instrumentos validados para este estudio. La revisión de la literatura se realizó en las bases de datos "Web o Science" (WOS), "Scorpus," y "Dialnet". Se utilizaron palabras clave tales como: "AFMN", "Educación Física (EF)", "Educación Secundaria Obligatoria (ESO)", "Percepción del profesorado", "Riesgos", "Accidentes", "Espeleología", "Escalada", "Senderismo" e "Innovación", filtrando los resultados dentro del periodo comprendido entre

Para citar este artículo utilice la siguiente referencia: Hurtado, M.; Sanabrias, D.; Sánchez, Mª .; Cachón, J.. (2020). Actividades Físicas en el Medio Natural. Incidencia en la formación del Profesorado y su enseñanza en la ESO. Sportis Sci J, 6 (1), 18-42. 


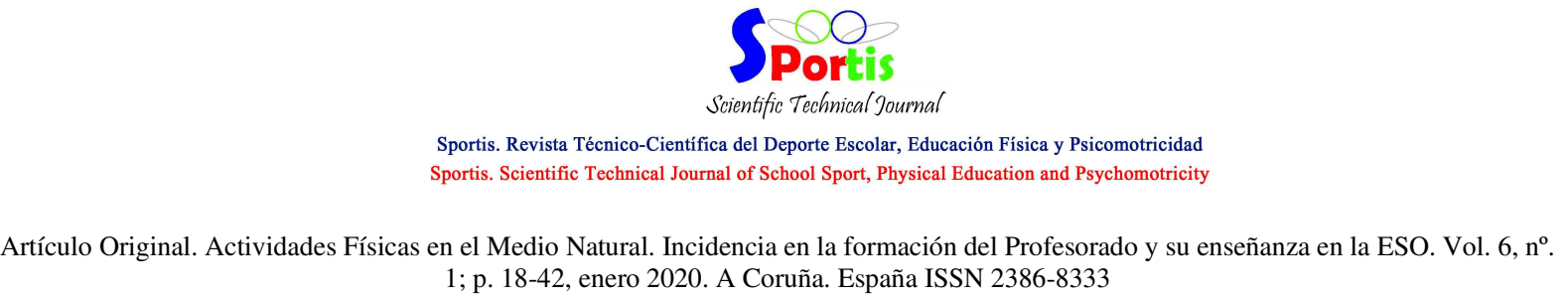
$1 ;$ p. 18-42, enero 2020. A Coruña. España ISSN 2386-8333

2010 y 2019. Al no aparecer cuestionarios en este sentido se ha realizado una entrevista individual semiestructurada.

Dicha entrevista se ha enviado a profesores de EF mediante la aplicación de "Google Drive: Google Forms" para aumentar la facilidad de difusión y se les ha avisado por teléfono, explicándoles así el modo de cumplimentación del cuestionario y la finalidad del estudio. Se han utilizado un total de 9 preguntas (Anexo 1). Finalmente, se obtuvo respuesta de 13 docentes.

\section{Análisis de resultados y Discusión}

\section{Los Planes de Estudio}

La mayor parte de docentes de EF en ESO son Licenciados o Graduados en Ciencias de la AF y el Deporte (CCAFYD),porque la docencia es la principal actividad profesional de las personas tituladas universitarias en CCAFYD (37.7\%) según el informe publicado por el Consejo General de Colegios Profesionales de la EF y del Deporte "Situación del mercado laboral de las personas tituladas universitarias en CCAFYD (2019)".Por lo que cabe realizar una revisión de los planes de estudio con el fin de aclarar el grado de conocimiento que poseen dichos titulados en referencia a las AFMN, comenzando por la relación de asignaturas en los Grados impartidos en las Universidades Andaluzas (Gráfico 1).

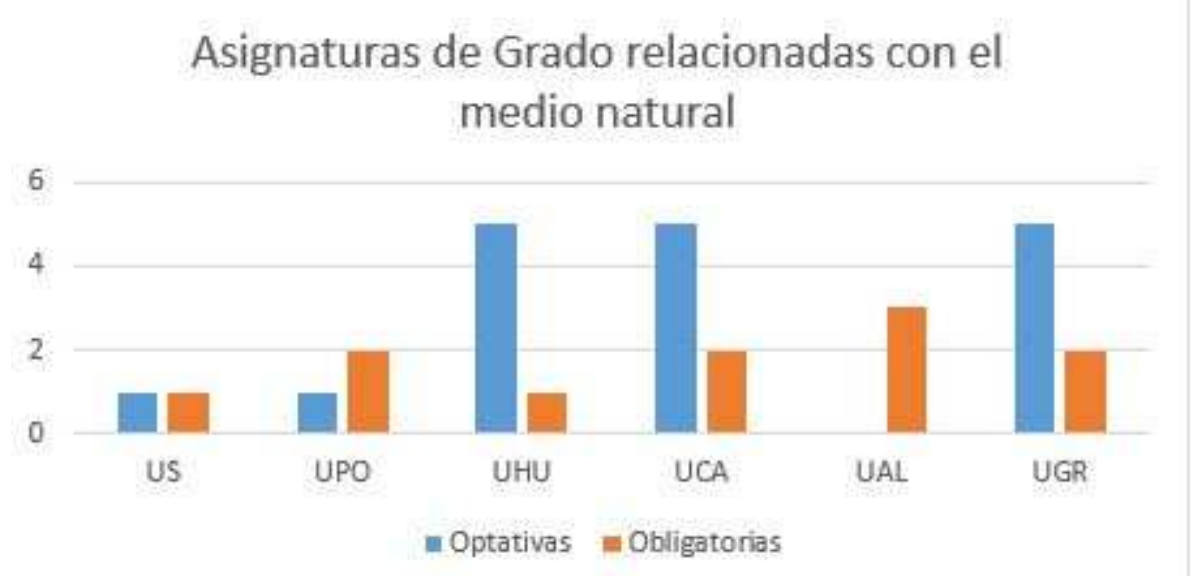

Gráfico 1: $\mathrm{N}^{\mathrm{o}}$ asignaturas de Grado en Ciencias Actividad Física y Deporte relacionadas con el Medio

Para citar este artículo utilice la siguiente referencia: Hurtado, M.; Sanabrias, D.; Sánchez, Mª .; Cachón, J.. (2020). Actividades Físicas en el Medio Natural. Incidencia en la formación del Profesorado y su enseñanza en la ESO. Sportis Sci J, 6 (1), 18-42. 
Artículo Original. Actividades Físicas en el Medio Natural. Incidencia en la formación del Profesorado y su enseñanza en la ESO. Vol. 6, n . 1 ; p. 18-42, enero 2020. A Coruña. España ISSN 2386-8333

Analizando los planes de estudio publicados en la web de las diferentes universidades públicas andaluzas donde se imparte el Grado en CCAFYD, de las 283 asignaturas que se ofertan solo 28 (9.9\%) están relacionadas con la AFMN, de las cuales 17 se ofertan como asignaturas optativas y 11 como obligatorias. En esta clasificación se incluyen asignaturas relacionadas con la gestión de actividades en el Medio Natural como por ejemplo "Creación y Gestión de Empresas de Turismo Activo (Universidad de Huelva)" o "Dinamización y Recreación: Sector Turístico y Espacios Naturales (Universidad de Cádiz)”.
\end{abstract}

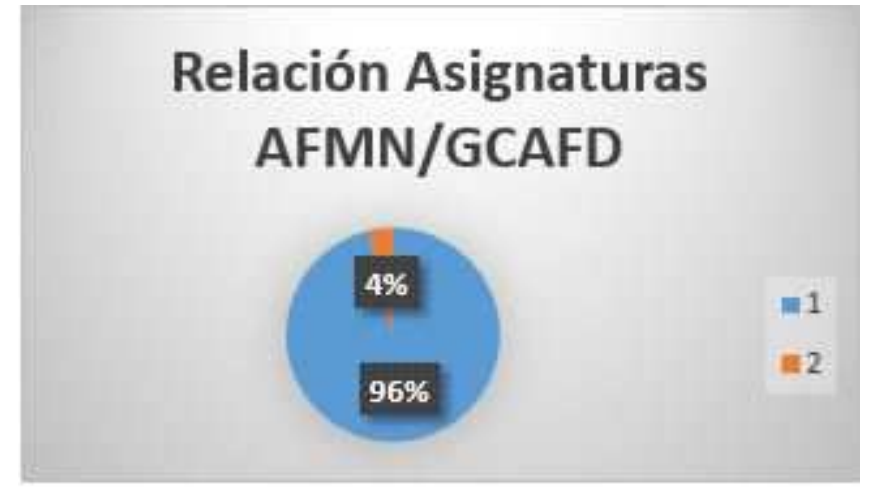

Gráfico 2: Relación asignaturas de Grado con asignaturas obligatorias relacionadas con AFMN

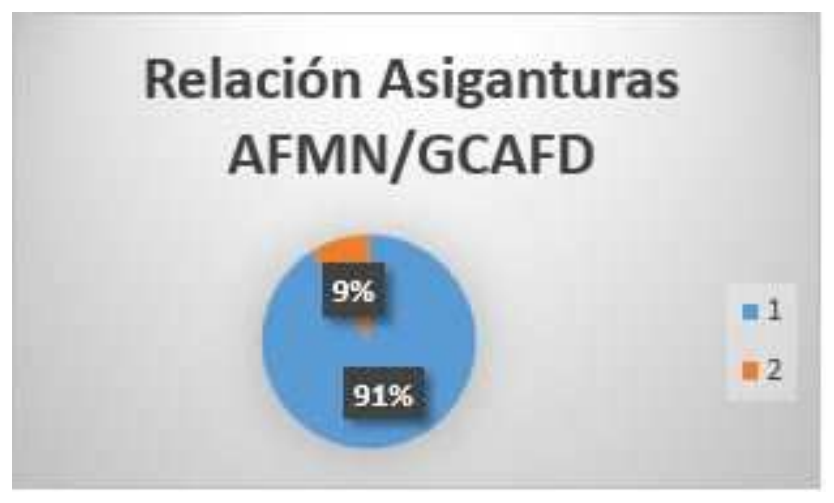

Gráfico 3: Relación asignaturas de Grado con asignaturas optativas relacionadas con AFMN

Se podría hablar del $9.9 \%$ de asignaturas relacionadas con el Medio Natural siempre y cuando, el alumnado haya optado por cursar todas las optativas de esta rama, sin embargo, si opta por otras asignaturas el porcentaje baja hasta el 3.8\% (Gráfico 2 y 3). Estos datos se alcanzarían realizando la media de todas las universidades, pero si nos centramos en la que menos y la que más asignaturas obligatorias ofertan, los datos revelan que en la Universidad de Sevilla los estudiantes obtendrían el grado habiendo cursado 1 asignatura de este ámbito (2.5\%), frente a la Universidad de Almería, donde consiguen el titulo tras superar 3 asignaturas relacionadas con la $\operatorname{AFMN}(7.5 \%)$. 


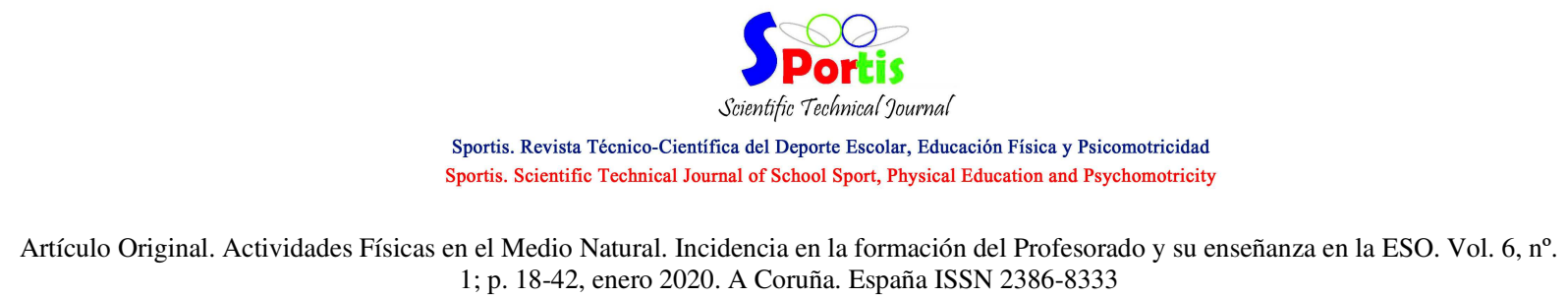

1; p. 18-42, enero 2020. A Coruña. España ISSN 2386-8333

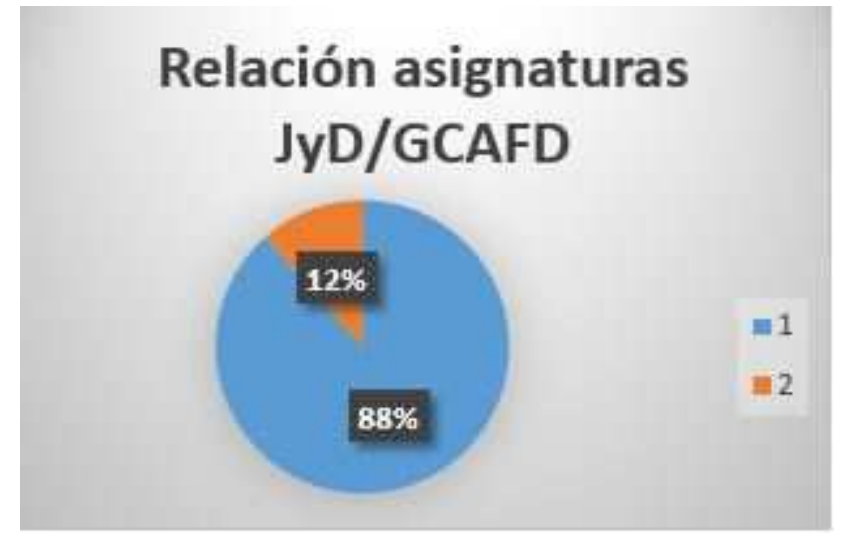

Gráfico 4: Relación asignaturas de Grado con asignaturas obligatorias relacionadas con el bloque de

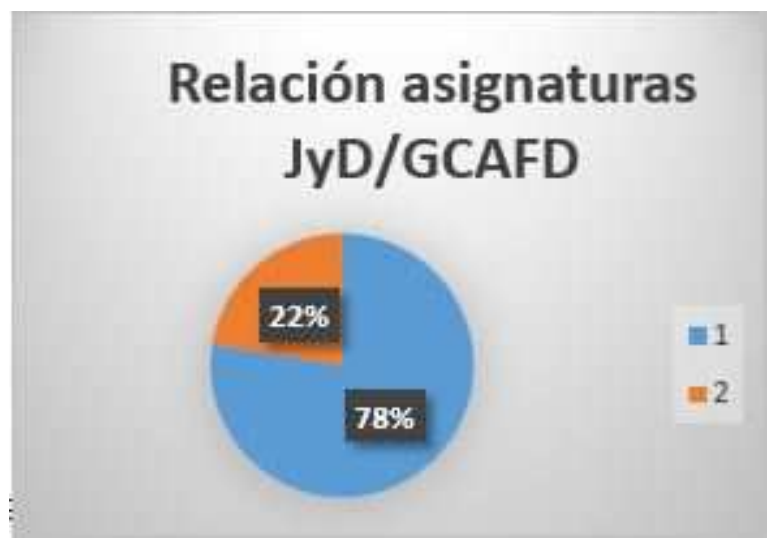

Gráfico 5: Relación asignaturas de Grado con asignaturas optativas relacionadas con el bloque de juegos

Comparando con asignaturas del bloque $3^{\circ}$ : Juegos y Deportes, se encuentra que en los planes de estudio se ofertan, entre todas las Universidades andaluzas, 82 materias (22\%), de las cuales 37 son obligatorias y 45 optativas. En esta clasificación se incluyen asignaturas como "Fundamentos de los Deportes de Raqueta (Universidad de Sevilla)", "Perfeccionamiento Deportivo: Balonmano (Universidad de Granada)" o "Enseñanza y Promoción del Voleibol (Universidad de Huelva)".

De igual modo que con el bloque AFMN, el 22\% se alcanzaría si el alumno opta por cursar todas las asignaturas de esta especialidad, sin embargo, si prepara asignaturas de diversas ramas, dicho porcentaje podría disminuir hasta el 12\% (Gráficos 4 y 5).

Estos datos revelan la escasa importancia que las instituciones educativas superiores otorgan a las AFMN, obteniendo, junto a la expresión corporal (Bloque $4^{\circ}$ ), unos índices de desarrollo en la formación del profesorado muy por debajo de otros bloques como el de Juegos y Deportes.

Lo expuesto podría explicar los resultados publicados por Cañadas, Castejón, \& Santos (2019), tras analizar el perfil profesional de 308 profesores universitarios, 490

Para citar este artículo utilice la siguiente referencia: Hurtado, M.; Sanabrias, D.; Sánchez, Mª ; Cachón, J.. (2020). Actividades Físicas en el Medio 


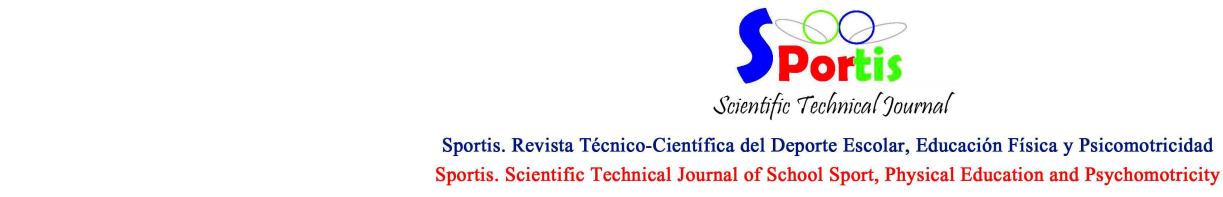

Artículo Original. Actividades Físicas en el Medio Natural. Incidencia en la formación del Profesorado y su enseñanza en la ESO. Vol. 6, nº 1 ; p. 18-42, enero 2020. A Coruña. España ISSN 2386-8333

egresados y 1.184 alumnos de los estudios de Magisterio con especialidad en EF y de CCAFD de 21 Universidades españolas. Los autores exponen que "llama la atención que ese conocimiento (el pedagógico) cuando se refiere a AFMN sea la puntuación más baja de todas". De igual forma, autores como Sáez-Padilla (2010) y Sáez-Padilla \& Fuentesal (2014), siguen la misma línea de crítica al sistema universitario argumentando que en los actuales planes de estudio la carga lectiva de AFMN es escasa.

Este trato discriminatorio se podría justificar con el hecho de que el Grado no solo da acceso a empleos de docencia o relacionados con las AFMN. Sin embargo, si se revisa el temario oficial de las oposiciones de acceso al cuerpo docente de ES por la especialidad de $\mathrm{EF}$, se puede observar que de los 65 temas solo 2 están directamente relacionados con este bloque, frente a los 17 de Condición física o los 8 de Juegos y deportes. Por lo que, al igual que en los planes de estudio del Grado y junto al bloque de Expresión corporal (3 temas) su presencia en la formación del profesorado es escasa.

De hecho, se podría decir que la falta de formación del profesorado en relación a la práctica de AFMN puede ser uno de los principales factores que justifiquen los datos obtenidos por Granero, Baena,\& Martínez Molina (2010), los cuales reflejan que las principales y prácticamente únicas actividades que se desarrollan dentro del bloque $5^{\circ}$ son, en primer lugar, la orientación, seguida muy de cerca por el senderismo y, por último, como actividad significativa, los juegos en la naturaleza y gimkanas. Datos que concuerdan con los obtenidos por Sáez-Padilla (2005) y Torres, Monleón, Sánchez, Torres, \& Aranda (2016).

\section{Otra normativa legal en la práctica de AFMN}

Por los motivos expuestos, procede revisar en qué situación se encuentra la responsabilidad civil del profesorado de EF al aplicar las AFMN.

A pesar de los escasos accidentes recogidos en el informe del GREIM (2014), es innegable que durante la práctica de AF en el Medio Natural el individuo asume una serie de riesgos objetivos que pueden desencadenar en un accidente. Sin embargo, estos riesgos no son exclusivos de la práctica de AFMN si no que son intrínsecos de la práctica físico deportiva y, por lo tanto, están presentes en todas las sesiones de EF. Por ello, el profesorado debe conocer

Para citar este artículo utilice la siguiente referencia: Hurtado, M.; Sanabrias, D.; Sánchez, Mª .; Cachón, J.. (2020). Actividades Físicas en el Medio 


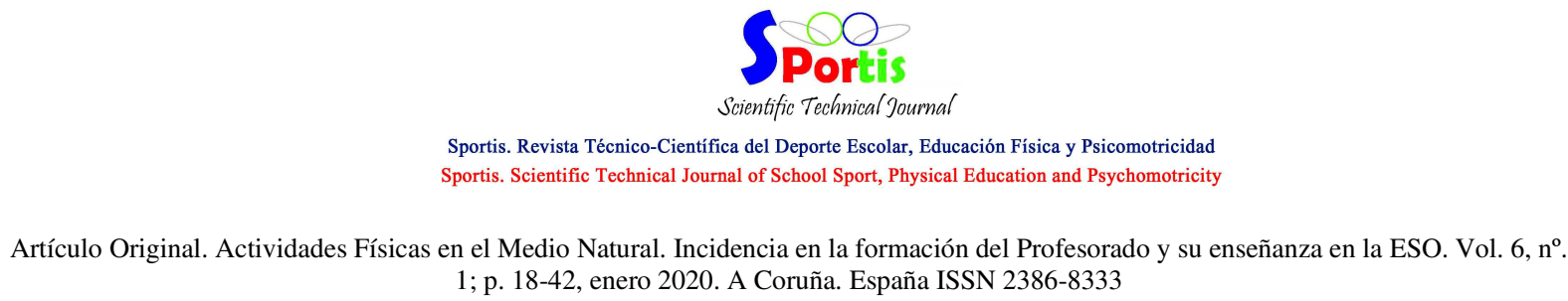

$1 ;$ p. 18-42, enero 2020. A Coruña. España ISSN 2386-8333

y asumir los inherentes riesgos que existen en las instalaciones deportivas de los centros educativos, así como, en algunos planteamientos didácticos, ya que según lo establecido en la Ley 50/1980 "habrá que reparar los daños y perjuicios que se produzcan a terceros". Sin embargo, dichos daños se repararán según el artículo 1902 del Código Civil "siempre que, por acción u omisión exista un comportamiento generador de daño, un resultado dañoso y un nexo causa" (Latorre, Mejías, Gallego, Muñoz, Santos, \& Adell, 2012).

En este sentido, la legislación obliga a los titulares de instalaciones deportivas, entre los que se encuentran todos los Institutos de ESO, a contratar una póliza de responsabilidad y asistencia sanitaria (Decreto 125/2008), que cubra el coste de los perjuicios y daños que puedan ocasionarse durante su utilización y práctica. Además, la Administración Pública está obligada a cubrir "los daños y perjuicios causados a los particulares a consecuencia del funcionamiento de los servicios públicos o de la actuación de sus funcionarios" (Ley 7/1985; Ley 30/1992; Ley 4/1999).

Silva (2015), realizó una revisión de las resoluciones judiciales y administrativas sobre accidentes producidos durante las sesiones de EF entre 1988 y 2012, y concluyó, que la mayoría de reclamaciones fueron desestimadas, aunque se encontraron casos donde el docente fue declarado culpable por "estar ausente de clase y por falta de prevención de las situaciones".

Desde el punto de vista pedagógico, Latorre \& Pérez Jiménez (2012) exponen lo que ya dijo Guillén (1999), que para evitar un accidente el docente de EF debe "prestar atención a los riesgos de la práctica físico-deportiva escolar desde varios puntos de vista", especialmente atendiendo a los alumnos, para quien es el profesor debe tener presente una posible descoordinación entre ellos, su condición física, deficiencias técnicas, sobre todo en temas relacionados con el Medio Natural; falta de concentración, desconfianza que le generen las actividades propuestas o la inmadurez y la intrepidez propias de los adolescentes.

Esta atención al alumnado se completa con la realización de autocrítica y conocimiento técnico de la actividad y/o una posible mala programación e inadecuación de las actividades propuestas causadas por una falta de secuenciación de los contenidos.

Para citar este artículo utilice la siguiente referencia: Hurtado, M.; Sanabrias, D.; Sánchez, Mª ; Cachón, J.. (2020). Actividades Físicas en el Medio Natural. Incidencia en la formación del Profesorado y su enseñanza en la ESO. Sportis Sci J, 6 (1), 18-42. 


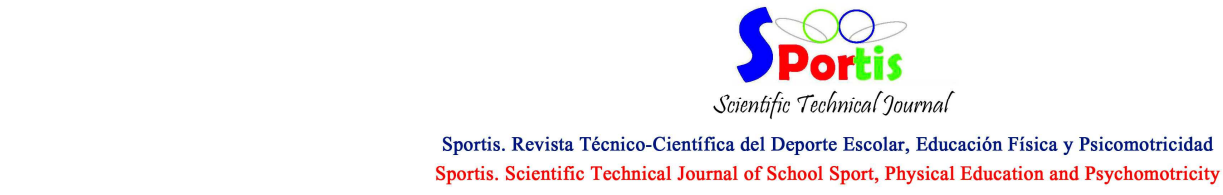

Artículo Original. Actividades Físicas en el Medio Natural. Incidencia en la formación del Profesorado y su enseñanza en la ESO. Vol. 6, nº 1 ; p. 18-42, enero 2020. A Coruña. España ISSN 2386-8333

Asimismo el profesor debe tener presentes las circunstancias o contextos propios de la actividad fisco-deportiva: estado de las instalaciones, ambiente que rodea a la actividad, climatología adversa e indumentaria deportiva.

Para reducir estos riesgos, Latorre \& Muñoz (2011) reflejan la importancia de la seguridad que debe existir en las clases de EF, insistiendo en la necesidad de tener en cuenta tanto la seguridad pasiva como la activa. Los autores definen la seguridad pasiva como aquella que se debe realizar antes de presentar la sesión, por ejemplo, cerciorarse de que las porterías están bien ancladas, que las canastas tengan un buen sistema antivuelco y en definitiva que se cumplan los estándares de calidad exigidas por la ley. Sin embargo, algunos autores aseguran que las instalaciones deportivas de los centros andaluces presentan una gran falta de seguridad, debido, sobre todo, a un incumplimiento de la normativa (Latorre, Mejía, \& Gallego, 2010; Lucio Morilla, 2002).

Latorre \& Muñoz (2011) definen la seguridad activa como la que engloba, por un lado, la percepción del riesgo que tienen los alumnos, y, por otro lado, la capacitación profesional del docente en temas de seguridad. La percepción del riesgo está relacionada con diversos factores psicológicos y de la personalidad como pueden ser experiencias previas, autocontrol, estrés emocional, autoconfianza. (Ivarsson \& Urban, 2010; Ortín, Montero, Garcés de los Fayos, \& Olmedilla, 2010).

Desde el punto de vista del docente hay que minimizar el riesgo al máximo en los ejercicios propuestos, por ejemplo, proteger la zona de caída si se realizan ejercicios en altura, recalcar el objetivo del ejercicio, así como hacer hincapié en las normas de seguridad (Latorre \& Pérez Jiménez, 2012). Por supuesto, cabe destacar que el docente, en función de licenciado o graduado en CCAFD, debería tener los conocimientos suficientes para realizar cualquier tipo de actividad que encuadre en el currículo de EF, conociendo las medidas de seguridad que se deben de llevar a cabo, así como la necesaria secuenciación de los contenidos y la adecuación de los mismos. Con el fin de que ni la falta de condición física, ni las deficiencias técnicas, ni la inmadurez o falta de coordinación del alumnado sean un factor de riesgo durante la realización de una sesión de EF. Asimismo, el profesor debe cerciorarse de que sus alumnos cumplan rigurosamente las medidas de seguridad establecidas.

Para citar este artículo utilice la siguiente referencia: Hurtado, M.; Sanabrias, D.; Sánchez, Mª; Cachón, J.. (2020). Actividades Físicas en el Medio Natural. Incidencia en la formación del Profesorado y su enseñanza en la ESO. Sportis Sci J, 6 (1), 18-42.

DOI: https://doi.org/10.17979/sportis.2020.6.1.5769

http://revistas.udc.es/ 


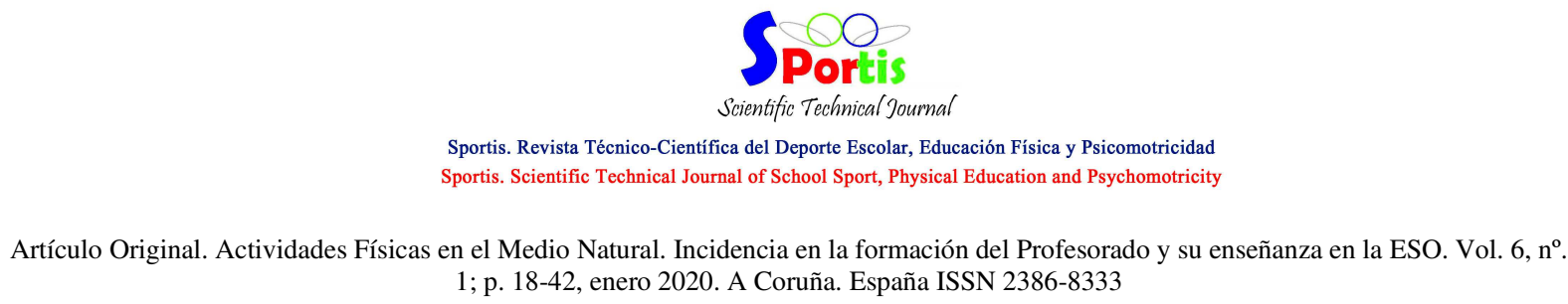

Aplicación de las AFMN

Aunque no se ha alcanzado la participación esperada, los datos obtenidos reflejan homogeneidad en cuanto al trato que recibe el bloque $5^{\circ}$ de contenidos por parte del profesorado.

A pesar de que la muestra obtenida (13 docentes) no se acerca a una población tradicionalmente significativa, existe una amplia diversidad en cuanto a la contextualización de los centros donde imparten docencia y, además, al tomar los datos por medio de una entrevista, permite considerarlos válidos. Por un lado, se ha alcanzado representación de todas las provincias andaluzas a excepción de Cádiz. Por otro lado, casi la mitad pertenece a zonas rurales y algo más de la mitad trabaja en centros ubicados en pequeñas y grandes ciudades. Sin embargo, la gran mayoría (8 de 13), tienen en común que realizan con alguno de sus cursos un viaje al centro de educación ambiental "Fuente del Roble" Arroyo Frío (Jaén).

Una vez contextualizada la muestra se tratarán cada una de las preguntas realizadas en la entrevista de forma individual (Anexo I).

Se pidió a los entrevistados que ordenasen los bloques de contenidos en función del peso que estos tenían en sus programaciones y, a excepción de un profesor, todos afirman que el bloque de contenidos de AFMN obtiene el último o penúltimo puesto, junto al bloque de Expresión Corporal. Datos que concuerdan con los obtenidos por Robles, Giménez, \& Abad (2010). Esto puede explicarse debido a que la formación exigida a los docentes, tanto en el grado como en la oposición, da una exposición casi nula tanto a los contenidos relacionados con la AFMN como a los relacionados con la expresión corporal.

Esta escasa exigencia formativa, se ve reflejada en las actividades que se trabajan en los centros educativos puesto que la gran mayoría de profesores practica exclusivamente senderismo y/u orientación en sus sesiones (Granero, Baena, \& Martínez Molina (2010), Sáez-Padilla, 2005) y Torres, Monleón, Sánchez, Torres, \& Aranda, 2016). Sobre ello los entrevistados responden:

1. "Dentro del centro hago una carrera de orientación y fuera hago una ruta de senderismo en la sierra" (Sujeto 2).

Para citar este artículo utilice la siguiente referencia: Hurtado, M.; Sanabrias, D.; Sánchez, Mª .; Cachón, J.. (2020). Actividades Físicas en el Medio Natural. Incidencia en la formación del Profesorado y su enseñanza en la ESO. Sportis Sci J, 6 (1), 18-42. DOI: https://doi.org/10.17979/sportis.2020.6.1.5769

http://revistas.udc.es/ 


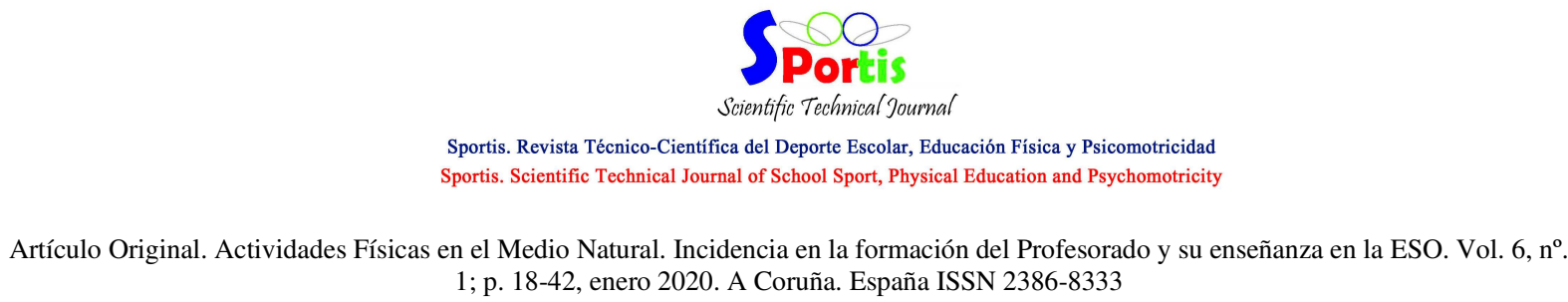

2. "Orientación en la naturaleza por medio de balizas, mapas y brújulas" (Sujeto 11).

3. "Con una UDI relacionada con el Medio Natural en la que se enseña la forma de orientarse con o sin brújula y con el mapa. Además, se realiza un sendero de bastante dureza para ellos. En otros cursos se hace una salida para realizar actividades propias del Medio Natural con una empresa de turismo activo" (Sujeto 10).

4. "Orientación en el centro y actividades puntuales para el resto de AF en los alrededores" (Sujeto 13).

Una minoría de profesores trabajan BTT -bicicleta todo terreno-), cabuyería, montaje de tiendas de campaña y piragüismo, según las siguientes respuestas:

1. “... $3^{\circ}$ ESO (semana verde en Cazorla FDR, BTT4 ESO (Orientación deportiva, piraguas) Bachillerato (orientación deportiva, BTT, piraguas)” (Sujeto 3).

2. “... con bachillerato también trabajo el montar tiendas de campaña y diversos refugios, con cuarto de las ESO cabuyería" (Sujeto 9).

Otra cuestión a tratar durante la entrevista, es la posibilidad de incorporar actividades como escalada, espeleología o barranquismo en sus sesiones dentro del centro educativo. La mayoría lo ve como algo positivo, aunque alguno de ellos lo considera inviable.

1. "Me parece muy buena idea siempre y cuando se disponga de los medios suficientes y se tenga experiencia en dichas actividades" (Sujeto 2).

2. “Necesarias para romper miedos y superación personal” (Sujeto 7).

3. "Siempre que se cumplan las medidas de seguridad pertinentes, me parecen muy adecuadas e interesantes, tanto por su carácter formativo, como por el añadido motivacional que conllevan" (Sujeto 8).

4. "Sería muy interesante poder introducir actividades, pero considero que el material sería muy complicado de conseguir. Los centros no tienen mucho poder adquisitivo y sería poco factible obtener material para esas actividades además de la carencia de conocimientos en muchas ocasiones por parte del profesorado" (Sujeto 9).

En cuanto a los motivos por los cuales el profesorado de EF rechaza en alguna medida la práctica de AFMN en sus clases, se puede decir que los principales motivos son la

Para citar este artículo utilice la siguiente referencia: Hurtado, M.; Sanabrias, D.; Sánchez, Mª .; Cachón, J.. (2020). Actividades Físicas en el Medio

Natural. Incidencia en la formación del Profesorado y su enseñanza en la ESO. Sportis Sci J, 6 (1), 18-42.

DOI: https://doi.org/10.17979/sportis.2020.6.1.5769

http://revistas.udc.es/ 


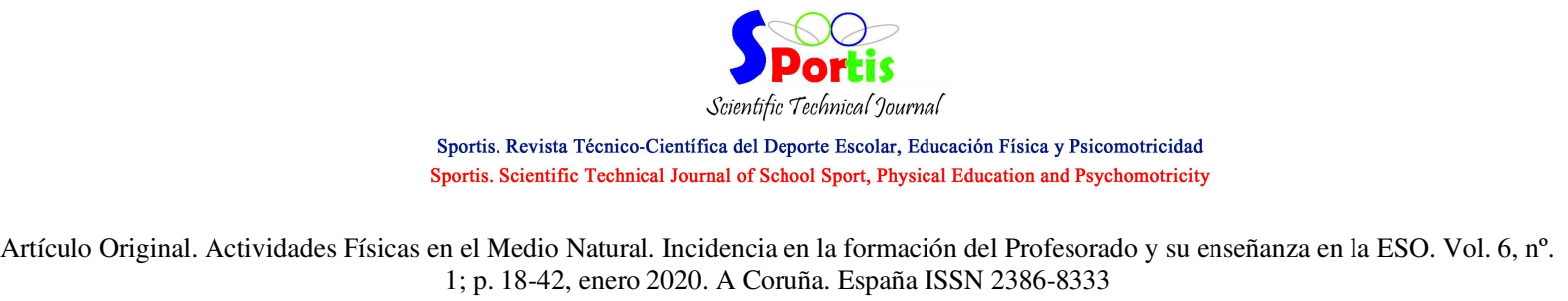

responsabilidad, la falta de experiencia, dificultad organizativa y los medios disponibles. Se expresa en frases como:

1. "Falta de medios y de experiencia" (Sujeto 2).

2. "Desconocimiento, falta de querer involucrarse, dificultad organizativa..." (Sujeto 8).

3. "Por qué son actividades qué son difícil de organizar y necesita mucho tiempo" (Sujeto 9).

4. "Problemas relacionados con la responsabilidad de salir del centro, falta de formación específica, implicación en horario extraescolar..." (Sujeto 1).

5. "Creo que por desconocimiento" (Sujeto 3).

6. "Falta de conocimiento, bagaje y acceso a este tipo de contenidos" (Sujeto 12).

7. "En las clases no se dispone de material adecuado como por ejemplo un rocódromo que cumpla con las normas de seguridad actuales. O cualquier otra instalación fija. En cuanto a orientación porque la preparación previa es muy ardua y no todo el profesorado esta actualizado" (Sujeto 13).

A pesar de ello, los datos revelan que todo el profesorado trabaja dicho bloque y realiza salidas, tal y como reflejan las respuestas a la pregunta de si realizan alguna salida al Medio Natural:

1. "Salidas a parque natural Marismas del Odiel. Ruta medio ambiental donde a través del senderismo se trabaja de manera interdisciplinar con otras áreas de conocimiento" (Sujeto $1)$.

2. "En todos los cursos. $1^{\circ}$ ESO senderismo y BTT. $2^{\circ}$ ESO senderismo y multi-aventura en Cazorla. $3^{\circ}$ ESO Orientación con plano sin brújula en parque periurbano "El Patriarca". $4^{\circ}$ ESO Escalada en top tope en zona Patriarca. 1 Bach Barranquismo semiacuático o acuático" (Sujeto 3).

3. "Si. Van a rocódromos, hacen salidas de aventura de varios días, kayak..." (Sujeto 6).

4. "Cazorla FDR, piragüismo en Río Genil, ruta senderismo y BTT, orientación deportiva...para el próximo año inclusión de semana azul y semana blanca” (sujeto 7).

Para citar este artículo utilice la siguiente referencia: Hurtado, M.; Sanabrias, D.; Sánchez, Ma .; Cachón, J.. (2020). Actividades Físicas en el Medio Natural. Incidencia en la formación del Profesorado y su enseñanza en la ESO. Sportis Sci J, 6 (1), 18-42. 
5. " $\mathrm{Si}$, en $1^{\mathrm{o}}$ de ESO se hace un sendero de unos $18 \mathrm{~km}$ por la sierra y actividades como tiro con arco, tirolina, etc. Con una empresa externa. En $2^{\circ}$ de ESO se realiza un viaje en el que se trabajan actividades como descenso de barrancos, kayak, orientación, etc. En $4^{\mathrm{o}}$ de la ESO se hace un sendero de 24km hacia el Santuario de Andújar" (Sujeto 8).

6. “1 ${ }^{\circ}$ ESO: Orientación en parque urbano. $2^{\circ}$ ESO: Día en el Medio Natural: circuito de BTT, rapel, tirolina, puentes monos, orientación. $3^{\circ}$ ESO: 3 días de convivencia en la sierra de Cazorla (descenso de cañones, vías ferrata, actividades acuáticas, tiro con arco, cerbatana, paintball, parque arbóreo. $4^{\circ}$ ESO: Semana de SKY en Andorra" (Sujeto 12).

Por último, se les preguntó sobre su opinión en cuanto a la formación recibida durante su etapa universitaria en lo referente a las AFMN. Hay respuestas muy diversas, sin embargo, se puede decir que más de la mitad (56\%) opina que fue escasa o mejorable:

1. "Escasa, y no capacita para trabajar en estos contenidos con cierta seguridad. Son experiencias, pero no formación" (Sujeto 1)

\section{2. "Muy escasa" (Sujeto 2)}

3. "Casi nula...tuve que ser autodidacta cuando tuve que dar los módulos de conducción de grupos en bicicleta y desplazamientos en Medio Natural en ciclo medio" (Sujeto 7)

4. "Escasa ya que tan solo obtuve una asignatura con este contenido y además las actividades prácticas fueron mediante un sorteo por lo que la oportunidad para realizar la fueron escasas" (Sujeto 9).

5. "Fue realmente mala" (Sujeto 12)

Frente al $44 \%$ que opina que su formación fue adecuada o correcta, los demás manifiestan:

1. "Creo que se da la formación necesaria para el desempeño de actividades en dicho medio, ya que existe una asignatura sobre actividades en el Medio Natural"' (Sujeto 5).

2. "Muy positiva. La tomaron como una parte importante para darnos a conocer actividades y lugares desconocidos para el alumnado, y promocionando su inclusión en nuestras vidas" (Sujeto 6).

Para citar este artículo utilice la siguiente referencia: Hurtado, M.; Sanabrias, D.; Sánchez, Ma .; Cachón, J.. (2020). Actividades Físicas en el Medio Natural. Incidencia en la formación del Profesorado y su enseñanza en la ESO. Sportis Sci J, 6 (1), 18-42.

DOI: https://doi.org/10.17979/sportis.2020.6.1.5769

http://revistas.udc.es/ 


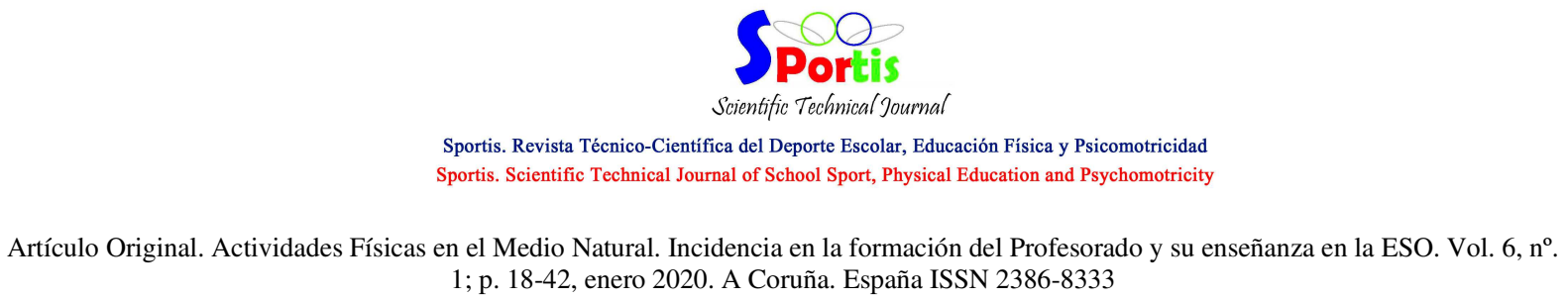

3. "Correcta, sin más" (sujeto 8).

4. "Pienso que fue una formación buena en contenidos teóricos y prácticos, con un profesorado experto y bien formado" (Sujeto 10).

5. "Adecuada, aunque era voluntaria" (Sujeto 13).

6. "Considero necesario que la formación mejore, sea más amplia, que los CEP ofrezcan formaciones de este tipo y que el profesorado luche porque esto sea así” (Sujeto 12).

\section{Conclusiones}

El bloque de contenidos $5^{\circ}$ de ESO: AFMN ha sufrido importantes cambios desde su incorporación al currículo desde la LOGSE (1990) hasta ahora. Con cada reforma educativa este bloque ha ido adquiriendo más importancia, sin embargo, frente a los múltiples beneficios que conlleva para el alumnado, tales como adquisición de valores y competencias clave, los datos muestran que es uno de los bloques que menos peso tiene en las programaciones de ESO.

Aunque son muchas y variadas actividades que engloban dicho bloque, el profesorado no las aplica, dedicándose fundamentalmente a senderismo y la orientación. La mayoría de docentes esgrime la dificultad de salir del centro, porque conlleva una ruptura del horario habitual de los escolares, así como a los problemas y dificultades organizativas propias de una salida al Medio Natural.

Respecto a cómo se trabaja el $5^{\circ}$ bloque de contenidos: AFMN en la etapa de ESO, para dar respuesta al objetivo 1: Muchos docentes de EF realizan salidas con diferentes empresas especializadas en AFMN, aplicando con ello los contenidos del bloque $5^{\circ}$ con su alumnado. Asimismo, la mayoría de profesores está a favor de la inclusión de actividades como la escalada, la espeleología o el barranquismo en sus sesiones, sin embargo no lo hacen.

Sobre las causas que llevan al profesorado a dar menor relevancia a estos contenidos en sus programaciones que da respuesta al objetivo 2: El profesorado no tiene el conocimiento necesario para introducir este tipo de actividades en los Institutos, ya que la formación recibida durante su etapa universitaria se centra en otros contenidos.

Para citar este artículo utilice la siguiente referencia: Hurtado, M.; Sanabrias, D.; Sánchez, Ma .; Cachón, J.. (2020). Actividades Físicas en el Medio

Natural. Incidencia en la formación del Profesorado y su enseñanza en la ESO. Sportis Sci J, 6 (1), 18-42.

DOI: https://doi.org/10.17979/sportis.2020.6.1.5769

http://revistas.udc.es/ 


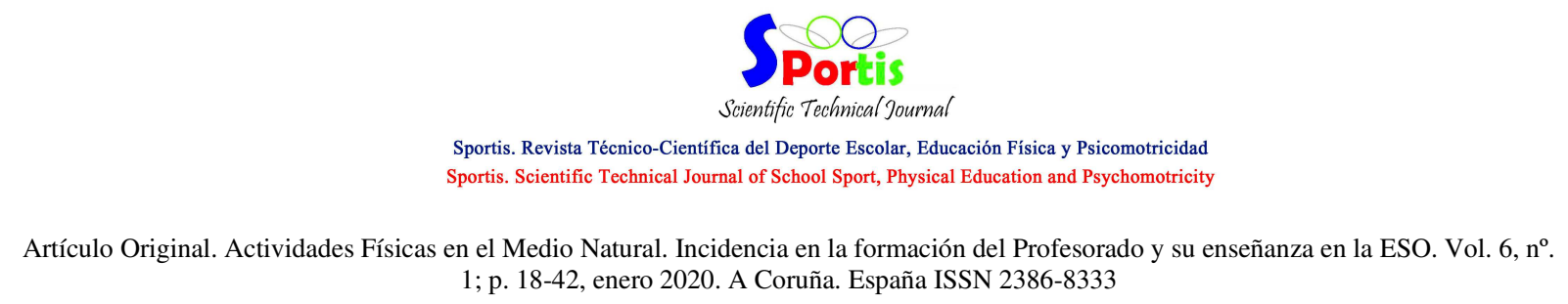

\section{Limitaciones y Mejoras}

Al tratarse de un estudio tan reducido, es susceptible y se recomienda su ampliación a más centros y a otras Comunidades Autónomas para mejorar las perspectivas. A tal efecto se adjuntan las preguntas aplicadas en el Anexo I.

Gran parte de la muestra realiza salidas a una única empresa de actividades en el Medio Natural, "Fuente del Roble" ubicada en Arroyo Frío (Jaén) lo que podría sesgar la información obtenida.

\section{Referencias bibliográficas}

Asociación Nacional de Empresas de Turismo Activo (2015), Informe sobre el Turismo activo en España 2014. Granada: ANETA

Baena-Extremera, A. \& Granero-Gallegos, A. (2014). Educación Física a través de la educación de aventura. Tándem, Didáctica de la EF, 45, 1-7.

Baena-Extremera, A. \& Granero-Gallegos, A. (2014). Estudio cuasi-experimental sobre actitudes de educación ambiental en EF. Cultura, Ciencia y Deporte, 25(9), 25-33. DOI: https://doi.org/10.12800/ccd.v9i25.386

Baena-Extremera, A. \& Granero-Gallegos, A. (2015). Efectos de las actividades en la naturaleza en la predicción de la satisfacción de la EF. Retos, 28, 9-14. DOI: https://doi.org/10.1590/1678-7153.201528202

Beltrán-Carrillo, V. J., \& Devís-Devís, J., \& Peiró-Velert, C. (2012). Actividad física y sedentarismo en adolescentes de la Comunidad Valenciana. Revista Internacional de Medicina y Ciencias de la Actividad Física y del Deporte, 12(45), 123-137.

Cañadas, L., Castejón, J., \& Santos-Pastor, M.L. (2019) Competencias docentes en la formación inicial del profesorado de educación física. Retos, 35, 284-288.

Castell, C. (2019). Naturaleza y salud: una alianza necesaria. Gaceta Sanitaria. In press. DOI: https://doi.org/10.1016/j.gaceta.2019.05.016

\footnotetext{
Para citar este artículo utilice la siguiente referencia: Hurtado, M.; Sanabrias, D.; Sánchez, Ma .; Cachón, J.. (2020). Actividades Físicas en el Medio Natural. Incidencia en la formación del Profesorado y su enseñanza en la ESO. Sportis Sci J, 6 (1), 18-42. DOI: https://doi.org/10.17979/sportis.2020.6.1.5769 http://revistas.udc.es/
} 
Artículo Original. Actividades Físicas en el Medio Natural. Incidencia en la formación del Profesorado y su enseñanza en la ESO. Vol. 6, nº $1 ;$ p. 18-42, enero 2020. A Coruña. España ISSN 2386-8333

Consejería de Educación (2016). Orden de 14 de julio, por la que se desarrolla el currículo correspondiente a la ESO en la Comunidad Autónoma de Andalucía, se regulan determinados aspectos de la atención a la diversidad y se establece la ordenación de la evaluación del proceso de aprendizaje del alumnado. Sevilla: BOJA 144 de 28/7.

Cuervo, C., Cachón, J., González, C., \& Zagalaz, M. L. (2017). Hábitos alimentarios y práctica de actividad física en una muestra de adolescentes de una ciudad del norte de España. Journal of Sport and Health Research, 9(1), 75-84.

Ewert, A. (1987). Outdoor adventure recreation: A trend analysis. Journal of Leisure Research, 5(2), 56-67.

Garrido-Méndez, A., Concha-Cisternas, Y., Rocha, F.P., Díaz-Martínez, X., Leiva, A.M., Troncosco, C.,...Celis-Morales, C. (2019). Influencia de la edad sobre el cumplimento de las recomendaciones de actividad física: resultados de la encuenta nacional de salud en Chile 2009-2010. Revista Chilena de Nutrición, 46(2), 121-128. DOI: https://doi.org/10.4067/s0717-75182019000200121

Gehris, J., Kress, J. \& Swalm, R. (2010). Students' Views on Physical Development and Physical Self-Concept in Adventure-Physical Education. Journal of Teaching in Physical Education, 29, 146-166. DOI: https://doi.org/10.1123/jtpe.29.2.146

Granero-Gallegos, A., Baena-Extremera, A., \& Martínez-Molina, M. (2010). Contenidos desarrollados mediante las actividades en el Medio Natural de las clases de Educación Física en secundaria obligatoria. Ágora, 12(3), 273-288.

Ivarsson, A. \& Urban, J. (2010). Psychological factors as predictors of injuries among senior soccer players. A prospectivestudy. Journal of Sports Science and Medicine, 9, 347352.

Latorre, P. A. \& Muñoz. A. (2011). Manual de control de la seguridad de los espacios y equipamientos deportivos de uso escolar. Jaén: Diputación de Jaén

Latorre, P. A. \& Pérez-Jiménez, M.I. (2012) Gestión de la seguridad en las actividades físicodeportivas escolares. EmásF, Revista Digital de EF, 3(18), 42-57.

Para citar este artículo utilice la siguiente referencia: Hurtado, M.; Sanabrias, D.; Sánchez, Mª .; Cachón, J.. (2020). Actividades Físicas en el Medio Natural. Incidencia en la formación del Profesorado y su enseñanza en la ESO. Sportis Sci J, 6 (1), 18-42.

DOI: https://doi.org/10.17979/sportis.2020.6.1.5769

http://revistas.udc.es/ 
Artículo Original. Actividades Físicas en el Medio Natural. Incidencia en la formación del Profesorado y su enseñanza en la ESO. Vol. 6, nº $1 ;$ p. 18-42, enero 2020. A Coruña. España ISSN 2386-8333

Latorre, P.A., Mejía, J.A., Gallego, M., Muñoz, A., Santos, M., \& Adell, M. (2012). Análisis de la seguridad de las instalaciones deportivas de las sedes de los juegos deportivos provinciales de Jaén. Journal of Sport and Health Research, 4(1), 57-66.

López, S., Maneiro, R., Moral, J. E., Amatria, M., Diez, P., Barcala, R., \& Abelairas, C. (2019). Los riesgos en la práctica de actividades en la naturaleza. La accidentabilidad en las prácticas deportivas y medidas preventivas. Retos, 36, 618-624.

López-Prado, J., Pardilla-Fernández Miranda, S., \& Álvarez-Gracia, D. (2013). Nuevos contenidos en educación física. Orientaciones didácticas para la integración del surf en el medio educativo. Revista Española de EF y Deportes, 402, 93-106.

Lucio-Morillas, M. (2003). Calidad y seguridad de las instalaciones y el material deportivo en los Centros de Educación Secundaria y Bachillerato de la provincia de Málaga. Tesis Doctoral. Málaga: Servicio de Publicaciones de la Universidad.

Méndez, A. \& Fernández-Río, J. (2011) Nuevas tendencias metodológicas en la enseñanza del esquí: orientaciones didácticas para su iniciación en los centros educativos. Apunts. EF $y \quad$ Deportes, 105, 35-43. DOI: https://doi.org/10.5672/apunts.20140983.es.(2011/3).105.04

Moral, J. E., Román-Palmero, J., López, S., Rosa, A., Pérez, J. J., \& García, E. (2019). Propiedades psicométricas de la Escala de Motivación Deportiva y análisis de la motivación en las clases de educación física y su relación con nivel de práctica de actividad física extraescolar. Retos, 36, 283-289.

OMS (2010). Recomendaciones mundiales sobre actividad física para la salud. Ginebra: OMS.

Ortín, F.J., Montero, E., Garcés de los Fayos, J., \& Olmedilla, A. (2010). Influencia de los factores psicológicos en las lesiones deportivas. Papeles del Psicólogo, 31(3), 281288.

Para citar este artículo utilice la siguiente referencia: Hurtado, M.; Sanabrias, D.; Sánchez, Mª .; Cachón, J.. (2020). Actividades Físicas en el Medio Natural. Incidencia en la formación del Profesorado y su enseñanza en la ESO. Sportis Sci J, 6 (1), 18-42. DOI: https://doi.org/10.17979/sportis.2020.6.1.5769

http://revistas.udc.es/ 
Artículo Original. Actividades Físicas en el Medio Natural. Incidencia en la formación del Profesorado y su enseñanza en la ESO. Vol. 6, nº 1 ; p. 18-42, enero 2020. A Coruña. España ISSN 2386-8333

Oviedo, G., Sánchez, J., Castro, R., Calvo, M., Sevilla, J. C., Iglesias, A., \& Guerra, M. (2013). Niveles de actividad física en población adolescente: estudio de caso. Retos, $23,43-47$.

Peñarrubia, C., Guillén, R., \& Lapetra, S. (2011). Las actividades en el Medio Natural en Educación Física. Valoración del profesorado de Secundaria sobre los principales factores de limitación para su desarrollo. Apunts. EF y Deportes, 104, 37-45. DOI: https://doi.org/10.5672/apunts.2014-0983.es.(2011/2).104.04

Peñarrubia, C., Guillén, R., \& Lapetra, S. (2013). Evolución de las actividades en el Medio Natural en EF a partir de 1990. Ágora, 15(2), 113-129.

Portell, M., Riba, M.D., \& Bayés, R. (1997). La definición de riesgo: implicaciones para su reducción. Revista de Psicología de la Salud, 9(1), 3-27.

Ramos, P., Jiménez-Iglesias, A., Rivera, F., \& Moreno, C. (2016). Evolución de la práctica de la actividad física en los adolescentes españoles. Revista Internacional de Medicina y Ciencias de la Actividad Física y del Deporte, 16(62), 335-353. DOI: https://doi.org/10.15366/rimcafd2016.62.010

Rincón, D., Arnal, J., Latorre, A., \& Sans, A. (1995). Técnicas de Investigación en Ciencias Sociales. Madrid: Dykinson.

Roberts, A., Hinds, J., \& Camic, P.M. (2019). Nature activities wellbeing in children and Young people: a systematic literatura review. Journal of Adventure Education and Outdoor Learning, 1-21. DOI: https://doi.org/10.1080/14729679.2019.1660195

Robles Rodríguez, J., Giménez Fuentes-Guerra, F.J., Abad Robles, M.T. (2010). Motivos que llevan a los profesores de EF a elegir los contenidos deportivos en la ESO. Retos, 18, $5-8$.

Rossi, M. L., Antún, M. C., Casagrande, M. L., Escasany, M., Ferrari, M. F., Raele, M. G., \& González, V. B. (2018). Evaluación de la intervención del programa Mi Escuela Saludable en una cohorte de escuelas que participaron durante 2016-2017. Revista de la Facultad de Ciencias Médicas, 76(1), 37-46.

Para citar este artículo utilice la siguiente referencia: Hurtado, M.; Sanabrias, D.; Sánchez, Mª .; Cachón, J.. (2020). Actividades Físicas en el Medio Natural. Incidencia en la formación del Profesorado y su enseñanza en la ESO. Sportis Sci J, 6 (1), 18-42. DOI: https://doi.org/10.17979/sportis.2020.6.1.5769 
Artículo Original. Actividades Físicas en el Medio Natural. Incidencia en la formación del Profesorado y su enseñanza en la ESO. Vol. 6, nº 1 ; p. 18-42, enero 2020. A Coruña. España ISSN 2386-8333

Ruiz, F., García, M.E., \& Piéron, M. (2009). Actividad física y estilos de vida saludables. Análisis de los determinantes de la práctica en adultos. Sevilla: Wanceulen.

Sáez-Padilla, J. \& Fuentesal, J. (2014). La formación universitaria en actividades físicodeportivas en el Medio Natural: situación actual y perspectivas de futuro. Tándem: Didáctica de la EF, 45, 14-21.

Sáez-Padilla, J. (2005). Las necesidades formativas del profesorado de educación física en la etapa de secundaria y su relación con las actividades en el Medio Natural. Revista Española de EF y Deportes, 3, 85-107.

Sáez-Padilla, J. (2010). La formación del profesorado de Educación Física de ESO en Andalucía en relación con las Actividades en el Medio Natural. Huelva: Servicio de Publicaciones de la Universidad.

Sáez-Padilla, J., Tornero-Quiñones, I., \& Sierra-Robles, A. (2017). La situación actual de la formación en actividades en el Medio Natural según el profesorado de Educación Física. Un estudio cualitativo con expertos en Andalucía. Espiral. Cuadernos del Profesorado, 10(21), 100-117. DOI: https://doi.org/10.25115/ecp.v10i21.1033

Sánchez-Hernández, A. (2017). Accidentes de montaña: siniestros, rescates y acciones preventivas de los deportes de montaña en España. Tesis doctoral. Universidad de Zaragoza.

Santos-Pastor, M.L., Cañadas, L., \& Martínez-Muñoz, F. (2019). Escala sobre la actitud medio ambiental en actividades físicas en el medio natural. Apunts. Educación Física y Deportes, 137,43-55.

Silva-Piñeiro, R. (2015). Responsabilidad del profesor de Educación Física: consecuencias de las reclamaciones legales de accidentes. Avances en supervisión educativa, 24, 1-17. DOI: https://doi.org/10.23824/ase.v0i24.10.

Slovic, P. (1987) Perception of Risk. Science, 236, 280-285. Recuperado de García Rodríguez, Y. (2009). Análisis y evaluación de la incidencia de la percepción de 
Artículo Original. Actividades Físicas en el Medio Natural. Incidencia en la formación del Profesorado y su enseñanza en la ESO. Vol. 6, nº $1 ;$ p. 18-42, enero 2020. A Coruña. España ISSN 2386-8333

riesgos en las conductas de seguridad en el ámbito laboral. Referencia del proyecto: UCM / RISKS. Universidad Complutense de Madrid.

Tárrega, J., Alguacil, M., \& Parra, D. (2018). Análisis de la Motivación Hacia la Práctica de Actividad Física Extraescolar en Educación Física. Multidisciplinary Journal of Educational Research, 8(3), 259-280. DOI: http://doi.org/10.17583/remie.2018.3754

Torres, J. F., Monleón, C., Sánchez, V., Torres, M.A., \& Aranda, P. (2016). AFMN en el área de Educación Física en centros de secundaria de la comarca de la Costera: análisis y propuesta didáctica. Actividad Física y Deporte: Ciencia y Profesión, 2(25), 31-43.

Villolota-Valverde, S. (2015). Accidentabilidad en montaña. Estadística de rescates en España y campañas de prevención. Tesis doctoral. Madrid: Universidad Camilo José Cela.

Wishart, L. \& Rouse, E. (2018). Pedagogies of outdoor spaces: an early chilhood educator profesional learning journey. Early Child Development and Care, 1-15. DOI: https://doi.org/10.1080/03004430.2018.1450250

\section{Anexo I. Preguntas de la entrevista semiestructurada.}

1. Explique brevemente el contexto de su centro (Zona rural o urbana, cercanía al Medio Natural, nivel socioeconómico del alumnado, etc.).

2. ¿Podría ordenar los bloques de contenidos en función del peso que tienen en su programación?

3. ¿Cómo trabaja el bloque de contenidos 5: Actividades físicas en el Medio Natural?

4. ¿Qué opina sobre el trabajo de este bloque a través de experiencias realizadas en salidas a empresas dedicadas al turismo activo?

5. ¿Qué opina sobre la inclusión de actividades como la escalada, el barranquismo o la espeleología dentro de las sesiones de Educación Física?

6. ¿Cuál cree que son los motivos por los cuales el profesorado de Educación Física siente cierta apatía a la práctica de actividades físicas en el Medio Natural dentro sus clases?

Para citar este artículo utilice la siguiente referencia: Hurtado, M.; Sanabrias, D.; Sánchez, Mª .; Cachón, J.. (2020). Actividades Físicas en el Medio

Natural. Incidencia en la formación del Profesorado y su enseñanza en la ESO. Sportis Sci J, 6 (1), 18-42.

DOI: https://doi.org/10.17979/sportis.2020.6.1.5769

http://revistas.udc.es/ 
Artículo Original. Actividades Físicas en el Medio Natural. Incidencia en la formación del Profesorado y su enseñanza en la ESO. Vol. 6, nº $1 ;$ p. 18-42, enero 2020. A Coruña. España ISSN 2386-8333

7. ¿En alguno de los cursos donde usted imparte docencia no se trabaja las actividades físicas en el Medio Natural?

8. ¿Realiza en alguno de los cursos alguna salida al Medio Natural? Explíquela brevemente.

9. ¿Cuál es su opinión sobre la formación recibida, durante su etapa universitaria, en lo referente a las actividades físicas en el Medio Natural? 